TRANSCRIPTION

\section{Competing forces}

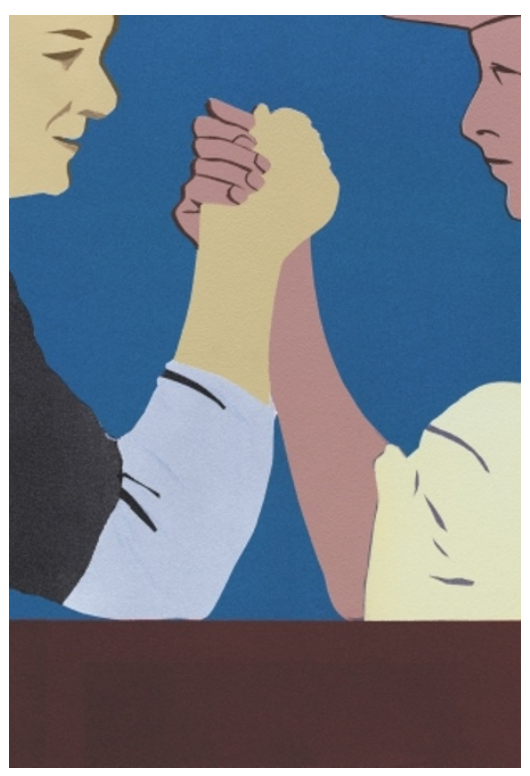

Chromatin structure has long been suspected to have a role in regulating cell fate but there has been no convincing evidence for this. However, data published in Molecular Cell now support a model whereby transcription factors and epigenetic silencing are integrated quantitatively to control cell differentiation.

Hutchins et al. used helper-T-cell $\left(\mathrm{T}_{\mathrm{H}}\right.$ cell) differentiation as a model system, and analysed $\mathrm{T}_{\mathrm{H}}$ cells from mice deficient in methyl-CpG-binding-domain protein 2 (Mbd2), one of the molecules that is thought to link DNA methylation and chromatinbased silencing.

In this study, cytokine signalling was manipulated to generate polarized $\mathrm{T}_{\mathrm{H} 1}$ and $\mathrm{T}_{\mathrm{H} 2}$ cells from uncommitted cells. The researchers found that, in Mbd2-deficient cells, the normal pattern of T-cell differentiation was dysregulated. For example, Mbd2-deficient cells produced ectopic interleukin (IL)-4 under $\mathrm{T}_{\mathrm{H} 1}$-polarizing conditions, and the normally essential transcriptional activator Gata-3 was dispensable for IL-4 induction. Moreover, expression of the signature genes encoding IL- 4 and interferon- $\gamma$ was increased in $\mathrm{T}_{\mathrm{H} 2}$ and $\mathrm{T}_{\mathrm{H} 1}$ cells, respectively, suggesting that Mbd2 might be crucial in silencing these cytokine genes during $\mathrm{T}_{\mathrm{H}}$-cell differentiation.

To investigate the relationships between both Gata- 3 and Mbd2, and the IL-4 gene, the authors determined relative Gata-3 messenger-RNA levels using the reverse-transcription polymerase chain reaction. Gata-3 mRNA levels were repressed as normal in cells cultured under $\mathrm{T}_{\mathrm{H} 1}$ conditions regardless of the $M b d 2$ genotype, indicating that it is not an excess of
Gata-3 that induces $I L-4$ in $M b d 2-$ mutant $\mathrm{T}_{\mathrm{H} 1}$ cells. In line with this observation, the authors proposed a quantitative competition model for activators (such as Gata-3) and silencers (such as Mbd2) whereby each regulator has an independent quantitative effect.

To test this hypothesis, cells with three different $M b d 2$ gene doses $\left(\mathrm{Mbd2}^{+/+}, \mathrm{Mbd2}^{+/-}, \mathrm{Mbd2^{-/- }}\right)$ were cultured under $\mathrm{T}_{\mathrm{H} 1}$ conditions (that is, where Gata-3 is not produced) and transduced with a bicistronic retrovirus encoding Gata-3 and green fluorescent protein (GFP). Cells with different levels of Gata-3 were evaluated based on the level of green fluorescence intensity. Increasing levels of Gata-3 rendered cells competent for IL-4 production in a manner that is inversely correlated with $M b d 2$ gene dosage.

To investigate the basis for the competition between Gata-3 and Mbd2 further, the authors performed

\section{CELL CYCLE}

\section{Cyclin' around}

Cyclin destruction and the associated drop in cyclin-dependent kinase (CDK) activity are necessary for mitotic exit. In yeast, degradation of the S-phase cyclin Clb5 was thought to be essential, but a study reported in Nature now raises doubts about the importance of $\mathrm{Clb} 5$ in regulating mitotic exit.

In frog embryos, a single oscillator can manage the cell cycle. This negativefeedback oscillator alternates between $S$ and $M$ phases, and involves the regulation of a ubiquitin-protein ligase called the

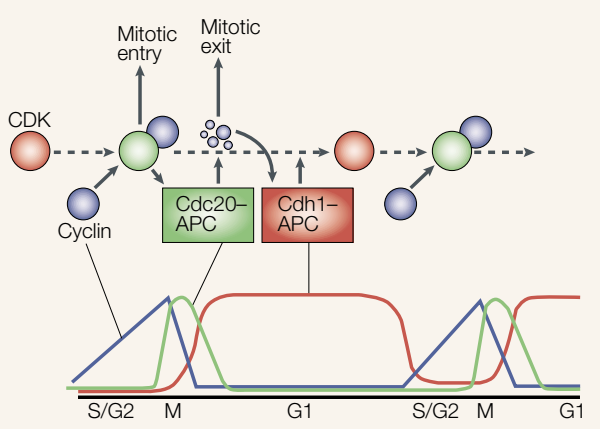

C (2002) Macmillan Magazines Ltd. anaphase-promoting complex (APC). This is activated by a molecule called Cdc20, which then recruits cyclins. CDKs phosphorylate and thereby activate Cdc20-APC, which triggers cyclin destruction and mitotic exit. As a result, the CDK activity decreases, which causes APC inactivation and re-accumulation of cyclin.

The cell cycle in somatic cells and yeast, however, is more complex because it includes a G1 phase that is important for cell growth and differentiation. G1 regulation might be achieved by a second oscillator that differs from the first one in using the APC-regulatory subunit Cdh 1 instead of Cdc20. CDKs inhibit Cdh1-APC, which means that, in late mitosis, $\mathrm{CDK}$ proteolysis causes activation of Cdh1-APC, which then maintains cyclin destruction throughout G1 (see diagram).

To address the question of whether the Clb5 cyclin must be destroyed, Wäsch and Cross constructed a yeast strain $(C L B 5 \Delta d b)$ in which the CLB5 gene lacked the destruction-box sequence that targets $\mathrm{Clb5}$ to Cdc20-APC. However, these cells did not seem to have any defect in mitotic exit, implying that proteolysis of $\mathrm{Clb} 5$ is not required. Exit also occurred in $C L B 5 \Delta d b$ cells that lacked Sic1, a CDK inhibitor responsible for further reducing CDK activity in late mitosis. This suggests that mitotic exit can progress without either Clb5 degradation or Sicl.

Next, Wäsch and Cross analysed the mitotic cyclin $\mathrm{Clb} 2$ as a potential Cdc20 target, and showed that $C L B 2 \triangle d b$ yeast cells were unable to exit mitosis. $\mathrm{Clb} 2$ might therefore be the cyclin that needs to be destroyed for mitotic exit to occur.

Previous studies had shown that cells that lacked Cdh1 and Sicl are not viable, suggesting that both proteins are essential for mitotic exit, and that the Cdh1-Sic1 oscillator might be the more important regulator. By contrast, Wäsch and Cross found that cells lacking these proteins do not have a marked defect in mitotic exit, and indeed survive, although poorly. However, the cells showed some abnormalities associated with an unstable G1 phase. This finding also highlights the important role of the Cdc20 oscillator in destroying $\mathrm{Clb} 2$.

So, the destruction of mitotic cyclins such as $\mathrm{Clb} 2$ is crucial to mitotic exit and the Cdc20 and Cdh1-Sic1 oscillators seem to work in a complementary manner, with the latter being important for a stable G1 phase.

$$
\text { Arianne Heinrichs }
$$

(2) References and links ORIGINAL RESEARCH PAPER Wäsch, R. \& Cross, F. R. APC-dependent proteolysis of the mitotic cyclin Clb2 is essential for mitotic exit. Nature 418, 556-562 (2002) FURTHER READING Morgan, D. O. \& Roberts J. M. Oscillation sensation. Nature 418, 495-496 (2002) 